\title{
Estrés en padres de recién nacidos prematuros de muy bajo peso hospitalizados en unidades de cuidados intensivos neonatales. Estudio multicéntrico
}

\author{
Stress in parents of very low birth weight preterm infants \\ hospitalized in neonatal intensive care units. A multicenter study
}

Lic. Francisca Wormald ${ }^{a}$, Dr. José L. Tapia ${ }^{a}$, Dra. Gabriela Torres ${ }^{b}$, Lic. Paula Cánepac, Lic. María Aurelia González d, Dra. Diana Rodrígueze, Dra. Marisol Escobarf, Lic. Bernardita Reyes ${ }^{a}$, Dra. Carola Capelli ${ }^{\ddagger}$, Lic. Laura Menéndez ${ }^{h}$,Dra. Patricia Delgado ${ }^{i}$, Dr. Sergio Treuer ${ }^{j}$, Dr. Rodrigo Ramírez ${ }^{k}$, Lic. Norma Borjal, M. Sc. Angélica Dominguez $z^{a}$ la Red Neonatal Neocosur

\section{RESUMEN}

Introducción. El nacimientode un hijo prematuro

a. División de

Pediatría, Escuela de Medicina, Pontificia Universidad Católica de Chile, Santiago, Chile.

b. Hospital Lagomaggiore, Argentina.

c. Hospital Dr. Sótero del Río, Chile.

d. Maternidad Sardá, Argentina.

e. Hospital Italiano de Buenos Aires, Argentina.

f. Hospital Guillermo Fricke, Chile.

g. Hospital Austral, Argentina.

h. Hospital J. A. Fernández, Argentina.

i. Hospital Cayetano Heredia, Perú.

j. Hospital Guillermo Grant, Chile.

k. Hospital Clínico Universidad de Chile, Chile.

1. Hospital de Clínicas CMI, Paraguay.

Correspondencia: Lic. Francisca Wormald: fwormald@gmail.com

Financiamiento:

Ninguno.

Conflicto de intereses:

Ninguno que declarar.

Recibido: 31-12-2014

Aceptado: 6-4-2015 es un evento estresante para sus padres. El objetivo de este estudio fue determinar el estrés inicial de padres de recién nacidos de muy bajo peso de nacimiento (RNMBPN) hospitalizados en 12 unidades de cuidadosintensivosneonatales en una red neonatal sudamericana, identificar los factores asociados y comparar el nivel de estrés parental en centros públicos vs. privados. Población y métodos. Estudio transversal en madres/padres de RNMBPN (de 500 a 1500 g). El estrés parental inicial se midió utilizando la Escala de Estrés Parental en una escala de 1 (bajo estrés) a 5 (alto estrés). Las características sociodemográficas de las madres/padres y de los neonatos fueron recolectadas y asociadas a los niveles de estrés parental.

Resultados. Participaron del estudio 273 padres / madres de un total de $218 \mathrm{RNMBPN}$. La encuesta fue aplicada en el 5,9 $\pm 2,0$ días de vida del recién nacido. El estrés parental total promedio fue de $3,1 \pm 0,8$, y la subescala rol parental fue aquella que puntuó más alto $(3,6)$. Tener un menor nivel educacional, estar desempleado, no haber tomado al recién nacido en brazos y el requerimiento de apoyo ventilatorio se asociaron a mayor estrés parental. El estrés fue mayor en madres que en padres y en centros públicos que en privados.

Conclusiones. En padres de RNMBPN, se encontró un estrés inicial moderado. El factor más relevante fue la alteración en su rol parental. El estrés parental fue mayor en las madres y en los centros públicos. Se requiere una mayor sensibilización, investigación e intervención en esta área.

Palabras clave: estrés psicológico, padres, recién nacido de muy bajo peso, unidades de cuidado intensivo neonatal.

http:/ /dx.doi.org/10.5546/aap.2015.303

\section{INTRODUCCIÓN}

Los recién nacidos de muy bajo peso de nacimiento (RNMBPN) deben enfrentar largas hospitalizaciones en unidades de cuidados intensivos neonatales (UCIN). En un estudio previo de nuestra Red, la estadía promedio de un RNMBPN fue de 59 días. ${ }^{1}$ Los padres de RNMBPN experimentan importantes niveles de estrés y tienen el desafío de adaptarse positivamente a esta experiencia. ${ }^{2-4}$

Diversos estudios han señalado que un alto nivel de estrés parental posterior al nacimiento de un hijo prematuro puede afectar el vínculo madre $/$ hijo $^{5}$ y el establecimiento de un apego seguro..$^{6-8}$ El estrés parental y los síntomas depresivos maternos han mostrado ser factores de riesgo para el futuro desarrollo social, conductual y funcional del recién nacido prematuro. ${ }^{9-11}$

Identificar los principales factores de riesgo asociado a estrés parental puede resultar útil para realizar intervenciones tempranas focalizadas, que aporten a la visualización del problema y a la sensibilización por parte del personal de salud que asiste a estos niños altamente vulnerables y a sus familias. No tenemos conocimiento de estudios multicéntricos sudamericanos que contemplen un número importante de participantes y que den cuenta de la realidad regional.

El objetivo principal de este estudio fue determinar el nivel de estrés inicial en padres de RNMBPN en las 
UCIN que pertenecen a la Red Sudamericana Neonatal Neocosur, identificar factores asociados y comparar el nivel de estrés parental en los centros públicos vs. los privados.

\section{POBLACIÓN Y MÉTODOS}

Estudio observacional, multicéntrico internacional y transversal realizado en unidades de neonatología pertenecientes a la Red Neocosur. ${ }^{12}$ Esta Red es una asociación voluntaria, sin fines de lucro, que reúne actualmente 24 UCIN de seis países de América del Sur (Argentina, Brasil, Chile, Paraguay, Perú, Uruguay).

Se hizo una invitación abierta a participar voluntariamente en este estudio a todos los centros, que al momento del inicio del estudio, sumaban 18.

Los criterios de inclusión fueron ser madre o padre de un RNMBPN hospitalizado en una UCIN, cuyo recién nacido (RN) tuviera una edad entre el tercer y el décimo día de vida. Además, los padres debían haber estado con el RN en la UCIN al menos una vez y haber firmado el consentimiento informado.

Los criterios de exclusión fueron que el RN presentara un diagnóstico de genopatía y/o malformaciones congénitas mayores o una afección de extrema gravedad, de acuerdo con el médico tratante; que las madres o padres hubiesen tenido con anterioridad un hijo prematuro hospitalizado; $y$ /o que fueran padres de trillizos o más hijos.

\section{Escala de estrés}

Escala de Estrés Parental (Parental Stressor Scale: Neonatal Intensive Care Unit; PSS: NICU, por sus siglas en inglés): esta encuesta fue diseñada por Miles ${ }^{13}$ para determinar la autopercepción de estrés de los padres, asociado a tener un hijo hospitalizado en la UCIN. Es autoadministrada y consta de 34 ítems divididos en tres subescalas, que reflejan las percepciones parentales acerca del ambiente de la UCIN (qué factor o situación particular es percibido, interpretado y representado cognitivamente como más estresante para los padres): a) Las vistas y sonidos del ambiente de la UCIN; b) El aspecto y comportamiento del RN (estado de enfermedad y los cuidados que requiere el niño); c) La alteración del rol parental (interacción con su hijo).

El encuestado debió puntuar entre 1 y 5 cada ítem, marcando 1 , cuando lo descrito en el ítem no había sido en absoluto estresante; 2 , cuando había sido poco estresante; 3 , moderadamente estresante; 4 , muy estresante; y 5 , extremadamente estresante. Se puntuaba 0 cuando el ítem descrito no aplicaba. El estrés parental total (EPT) corresponde al promedio de las tres subescalas. Se utilizó la versión validada en español de esta escala. ${ }^{14}$

La PSS: NICU ha demostrado tener una excelente validez y confiabilidad en estudios realizados en diferentes países. ${ }^{15-17}$

\section{Antecedentes demográficos y perinatales}

Se recolectaron los siguientes datos:

Antecedentes de embarazo y parto: tipo de parto, gesta, hospitalización durante el embarazo, control de embarazo. Antecedentes sociales: edad, estado civil, nivel educacional, situación laboral. El nivel educacional se categorizó en $<\mathrm{o} \geq 8$ años, lo que corresponde en la región a educación básica completa. Antecedentes del RN: edad gestacional, peso de nacimiento, Apgar 1 y 5 , sexo, apoyo ventilatorio y días de vida al momento de la evaluación. Otros antecedentes significativos: haber tomado en brazos al RN, antecedentes de abortos/mortinato. Además, se categorizó por centro público/privado.

La primera fase del estudio contempló una capacitación y evaluación piloto en cada unidad, que consistió en la aplicación del instrumento a 3 padres y/o madres a fin de pesquisar eventuales dificultades en la aplicación y/o comprensión de la escala.

La encuesta fue realizada en un espacio privado (fuera de la UCIN) y aplicada por un integrante del equipo de salud (médico, enfermero o psicólogo) que no fuera parte del equipo tratante del prematuro en ese momento. A su vez, se recogieron datos sociodemográficos y del historial de embarazo/parto. La aplicación de los instrumentos demoró alrededor de 30 minutos. Cada centro envió electrónicamente los datos obtenidos, que fueron llevados a una planilla central para luego ser analizados. Los datos se obtuvieron entre enero de 2010 y septiembre de 2011.

\section{Consideraciones éticas}

Este estudio fue aprobado por todos los comités de ética locales de los centros participantes.

\section{Análisis estadístico}

Se calculó el tamaño muestral necesario de 160 sujetos (80 en cada grupo) para estimar una diferencia entre dos promedios de un 
$15 \%(0,45$ puntos de diferencia) en la escala de estrés y asumiendo un $\mathrm{DE} \pm 1$ en la escala para cada grupo. Dado que se trataba de un estudio multicéntrico, en el que interesaba una mayor representatividad, se planeó un tamaño muestral más amplio, no inferior a 250, lo cual era suficiente para detectar las diferencias mencionadas y para estimar el estrés promedio global.

Se describió la población de padres/madres y de RN según sus características principales. Las variables continuas se reportaron con medias y desviación estándar y las categóricas, como frecuencias y porcentajes.

Para comparar el nivel promedio de estrés según las variables demográficas categóricas del RN, se usaron pruebas t Student o ANOVA para muestras independientes, de acuerdo con el número de niveles de variables categóricas. En este último caso, se utilizó la corrección de Bonferroni o Scheffé para comparaciones múltiples. Para contrastar variables continuas con el nivel de estrés, se utilizó la correlación de Pearson.

Para determinar un aumento en el riesgo, se utilizaron los OR (Odds Ratios) con un $95 \%$ de intervalo de confianza (95\% IC) mediante una regresión logística simple. El nivel de estrés fue categorizado en bajo $(\leq 3)$ y alto $(>3)$, concordante a lo empleado en un estudio previo. ${ }^{18}$

Se consideraron significativos valores $p$ inferiores a 0,05 . Los análisis estadísticos fueron realizados con SPSS 16.0.

\section{RESULTADOS}

Participaron 12 unidades de neonatología de cuatro países (Argentina, Chile, Paraguay, Perú) que forman parte de la Red Neocosur. Se entrevistaron un total de 273 padres/madres que tenían a sus hijos prematuros de 500 a $1500 \mathrm{~g}$ hospitalizados en la UCIN, a los 5,9 $\pm 2,0$ días de vida (media $\pm \mathrm{DE}$ ).

De las madres entrevistadas, un $74 \%$ había tenido parto por cesárea y un $61 \%$ había estado hospitalizada durante su embarazo actual. Al momento de la evaluación, el $40 \%$ de los padres había tomado a su hijo en brazos, al menos, una vez.

En cuanto al peso de los RN ( $n=218)$, un 33\% tenían un peso de nacimiento menor de $1000 \mathrm{~g}$. Respecto del apoyo ventilatorio que recibían los prematuros al momento de la evaluación, un $56 \%$ estaba con algún tipo de apoyo. De ellos, un $26 \%$ estaba conectado a ventilación mecánica; un $23 \%$, a presión positiva continua en la vía aérea (continuous positive airway pressure; $\mathrm{CPAP}$, por sus siglas en inglés); y un 7\%, con cánula nasal.

Las características generales de los padres entrevistados y sus RN aparecen en la Tabla 1. El EPT y los puntajes de las subescalas aparecen en la Tabla 2.

La alteración del rol parental tuvo el puntaje más alto dentro de las subescalas. Dentro de esta, los ítems que puntuaron más alto fueron estar separado de su hijo $(4,2 \pm 1,1)$, seguido de no poder alimentarlo $(3,8 \pm 1,4)$ y no ser capaz de protegerlo del dolor y de los procedimientos dolorosos $(3,8 \pm 1,5)$. Para la subescala aspecto y comportamiento, lo más estresante para los padres fue ver tubos y equipos cerca de su hijo o puestos en él $(3,3 \pm 1,4)$, seguido de ver agujas y tubos puestos en su hijo $(3,3 \pm 1,5)$ y la apariencia débil de su hijo $(3,1 \pm 1,5)$. Finalmente, en la subescala de vistas y sonidos, el ítem más alto de estrés parental fueron los sonidos repentinos

TABLA 1. Características demográficas de los padres y sus recién nacidos

\begin{tabular}{|c|c|c|}
\hline \multicolumn{2}{|l|}{ Padres } & \multirow{2}{*}{$\begin{array}{c}(\mathbf{n}=\mathbf{2 7 3}) \\
72,5\end{array}$} \\
\hline Sexo & Femenino (\%) & \\
\hline Edad & Años (media, DE) & $29,0(8,1)$ \\
\hline & Menor de 19 años (\%) & 9,2 \\
\hline Estado civil & Soltero/a (\%) & 32 \\
\hline Estado laboral & Desempleado/a (\%) & 43 \\
\hline Nivel educacional & $<8$ años $(\%)$ & 36 \\
\hline Recién nacido & & $(n=218)$ \\
\hline Peso de nacimiento (g) (media, DE) & $1135(268)$ & \\
\hline Edad gestacional (semanas) (media, DE) & $29(2,6)$ & \\
\hline Sexo femenino (\%) & 48,6 & \\
\hline Apgar 5 min (media, DE) & $8(1,6)$ & \\
\hline Apoyo ventilatorio (\%) & 56 & \\
\hline
\end{tabular}

DE: desvío estándar. 
de las alarmas $(3,4 \pm 1,4)$, seguido del ruido constante de los monitores y equipos $(2,7 \pm 1,4)$ y la presencia de monitores y equipos en la UCIN $(2,6 \pm 1,3)$.

El EPT fue más alto en las madres que en los padres $(p=0,011)$; a su vez, las madres mostraron tres veces mayor riesgo de estar altamente estresadas frente al contexto de la UCIN que los padres (OR $=3,35 ; 95 \%$ IC: $1,50-7,47)$. Los padres desempleados o las dueñas de casa se asociaron con un nivel más alto de estrés ( $\mathrm{p}=$ $0,024)$. En cuanto al nivel educacional, los padres con educación menor de 8 años también mostraron un mayor nivel de estrés $(\mathrm{p}=0,034)$. No se encontraron asociaciones significativas entre EPT y paridad, historia de abortos y hospitalización durante el embarazo. Sin embargo, se encontró un mayor EPT en mujeres con embarazos no controlados $(p=0,041)$. Finalmente, aquellas madres/padres que no habían tomado a su hijo en brazos presentaban un nivel más alto de estrés $(p<0,001)$ y tenían el doble de riesgo de estar altamente estresados (OR=2,13; 95\% IC: 1,22-3,74).

En cuanto a las variables del RN, el requerimiento de apoyo ventilatorio se asoció a un mayor EPT $(p=0,02)$, tanto si el RN se

TABLA 2. Subescalas de estrés parental y estrés parental total en relación con las características de los padres y del recién nacido de muy bajo peso de nacimiento

\begin{tabular}{|c|c|c|c|c|c|}
\hline & & $\begin{array}{l}\text { Rol parental } \\
\text { Media (DE) }\end{array}$ & $\begin{array}{c}\text { Aspecto y comportamiento } \\
\text { Media (DE) }\end{array}$ & $\begin{array}{l}\text { Vistas y sonidos } \\
\text { Media (DE) }\end{array}$ & $\begin{array}{l}\text { Estrés parental total } \\
\text { Media }(\mathrm{DE})\end{array}$ \\
\hline Total & & $3,6(1,0)$ & $3,1(0,9)$ & $2,6(0,9)$ & $3,1(0,8)$ \\
\hline \multicolumn{6}{|l|}{ RN } \\
\hline Sexo & $\begin{array}{l}\text { Hombre } \\
\text { Mujer } \\
\text { Valor } p\end{array}$ & $\begin{array}{c}3,64 \\
3,64 \\
0,944\end{array}$ & $\begin{array}{c}3,17 \\
3,26 \\
0,484\end{array}$ & $\begin{array}{c}2,61 \\
2,63 \\
0,913\end{array}$ & $\begin{array}{c}3,14 \\
3,17 \\
0,764\end{array}$ \\
\hline Peso de nacimiento (g) & $\begin{array}{l}\leq 1000 \\
>1000 \\
\text { Valor } p\end{array}$ & $\begin{array}{c}3,69 \\
3,61 \\
0,526\end{array}$ & $\begin{array}{c}3,31 \\
3,15 \\
0,228\end{array}$ & $\begin{array}{c}2,60 \\
2,62 \\
0,892\end{array}$ & $\begin{array}{c}3,20 \\
3,13 \\
0,490\end{array}$ \\
\hline Apgar $1 \mathrm{~min}$ & $\begin{array}{c}\leq 3 \\
>3 \\
\text { Valor } p\end{array}$ & $\begin{array}{c}3,82 \\
3,60 \\
0,150\end{array}$ & $\begin{array}{c}3,43 \\
3,16 \\
0,141\end{array}$ & $\begin{array}{c}2,92 \\
2,56 \\
0,047\end{array}$ & $\begin{array}{c}3,39 \\
3,11 \\
0,055\end{array}$ \\
\hline Soporte respiratorio & $\begin{array}{c}\text { Sí } \\
\text { No } \\
\text { Valor } p\end{array}$ & $\begin{array}{c}3,75 \\
3,50 \\
0,050\end{array}$ & $\begin{array}{c}3,29 \\
3,11 \\
0,172\end{array}$ & $\begin{array}{c}2,75 \\
2,45 \\
0,016\end{array}$ & $\begin{array}{c}3,26 \\
3,02 \\
\mathbf{0 , 0 2 0}\end{array}$ \\
\hline Tipo de parto & $\begin{array}{l}\text { Cesárea } \\
\text { Vaginal } \\
\text { Valor } p\end{array}$ & $\begin{array}{c}3,64 \\
3,65 \\
0,906\end{array}$ & $\begin{array}{c}3,17 \\
3,33 \\
0,258\end{array}$ & $\begin{array}{c}2,55 \\
2,81 \\
0,071\end{array}$ & $\begin{array}{c}3,12 \\
3,27 \\
0,212\end{array}$ \\
\hline \multicolumn{6}{|l|}{ Padres } \\
\hline Tipo & $\begin{array}{l}\text { Padre } \\
\text { Madre } \\
\text { Valor } p\end{array}$ & $\begin{array}{c}3,10 \\
3,73 \\
0,001\end{array}$ & $\begin{array}{c}2,89 \\
3,26 \\
0,043\end{array}$ & $\begin{array}{c}2,49 \\
2,64 \\
0,416\end{array}$ & $\begin{array}{c}2,82 \\
3,21 \\
\mathbf{0 , 0 1 1}\end{array}$ \\
\hline Edad (años) & $\begin{array}{c}<18 \\
\geq 18 \\
\text { Valor } p\end{array}$ & $\begin{array}{c}3,56 \\
3,65 \\
0,664\end{array}$ & $\begin{array}{c}3,30 \\
3,20 \\
0,570\end{array}$ & $\begin{array}{c}2,87 \\
2,59 \\
0,176\end{array}$ & $\begin{array}{c}3,24 \\
3,15 \\
0,511\end{array}$ \\
\hline $\begin{array}{l}\text { Nivel educacional } \\
\text { (años) }\end{array}$ & $\begin{array}{l}<8 \\
\geq 8\end{array}$ & $\begin{array}{l}3,74 \\
3,57\end{array}$ & $\begin{array}{l}3,41 \\
3,08\end{array}$ & $\begin{array}{l}2,72 \\
2,55\end{array}$ & $\begin{array}{l}3,29 \\
3,06\end{array}$ \\
\hline & Valor $p$ & 0,185 & 0,010 & 0,192 & 0,034 \\
\hline Situación laboral & $\begin{array}{c}\text { Desempleado } \\
\text { Empleado } \\
\text { Valor } p\end{array}$ & $\begin{array}{c}3,72 \\
3,54 \\
0,186\end{array}$ & $\begin{array}{c}3,32 \\
3,08 \\
0,061\end{array}$ & $\begin{array}{c}2,76 \\
2,46 \\
\mathbf{0 , 0 2 0}\end{array}$ & $\begin{array}{c}\text { 3,27 } \\
\text { 3,03 } \\
\mathbf{0 , 0 2 4}\end{array}$ \\
\hline Tomar en brazos & $\begin{array}{c}\text { Sí } \\
\text { No } \\
\text { Valor p }\end{array}$ & $\begin{array}{c}3,38 \\
3,81 \\
\mathbf{0 , 0 0 1}\end{array}$ & $\begin{array}{c}2,99 \\
3,35 \\
0,004\end{array}$ & $\begin{array}{c}2,35 \\
2,79 \\
\mathbf{0 , 0 0 1}\end{array}$ & $\begin{array}{c}2,91 \\
3,32 \\
<0,001\end{array}$ \\
\hline Estado civil & $\begin{array}{c}\text { Casado/convive } \\
\text { Sin pareja } \\
\text { Valor } p\end{array}$ & $\begin{array}{c}3,75 \\
3,56 \\
0,156\end{array}$ & $\begin{array}{c}3,34 \\
3,15 \\
0,168\end{array}$ & $\begin{array}{c}2,66 \\
2,59 \\
0,606\end{array}$ & $\begin{array}{c}3,25 \\
3,10 \\
0,177\end{array}$ \\
\hline
\end{tabular}

RN: recién nacido; DE: desvío estándar. 
encontraba con apoyo de ventilación mecánica $(p=0,017)$ como con CPAP $(p<0,0001)$.

En relación con el tipo de centro, los padres atendidos en centros públicos $(n=165)$ mostraron un nivel mayor de estrés $(\mathrm{p}<0,001)$ que aquellos atendidos en los centros privados $(n=53)$ (Figura 1).

\section{DISCUSIÓN}

Nuestro estudio encontró un EPT inicial moderado $(3,1)$ en los padres de RNMBPN hospitalizados en las UCIN incluidas en esta investigación. Esto es similar a lo reportado en estudios recientes en el tema. ${ }^{18-20}$ La subescala con el puntaje promedio más alto para estrés fue la alteración en el rol parental, seguida por el aspecto y comportamiento del RN y las vistas y sonidos de la UCIN. Estos hallazgos son consistentes con otros estudios que han empleado la PSS: NICU ${ }^{19-22}$ y muestran que una importante causa de estrés en las familias es no poder satisfacer las necesidades básicas de su hijo/a, así como no poder protegerlos y tener que estar separados de ellos. ${ }^{23}$

Un reciente estudio de India ${ }^{22}$ muestra resultados similares a los nuestros. La subescala con mayor puntuación es la alteración del rol parental $(4,1)$, seguida del aspecto y comportamiento $(4,1)$ y las vistas y sonidos $(2,5)$. Además, un estudio multicéntrico italiano ${ }^{18}$ realizado antes del alta hospitalaria también

FiguRA 1. Estrés parental total (media, desvío estándar) de acuerdo con el centro público/privado

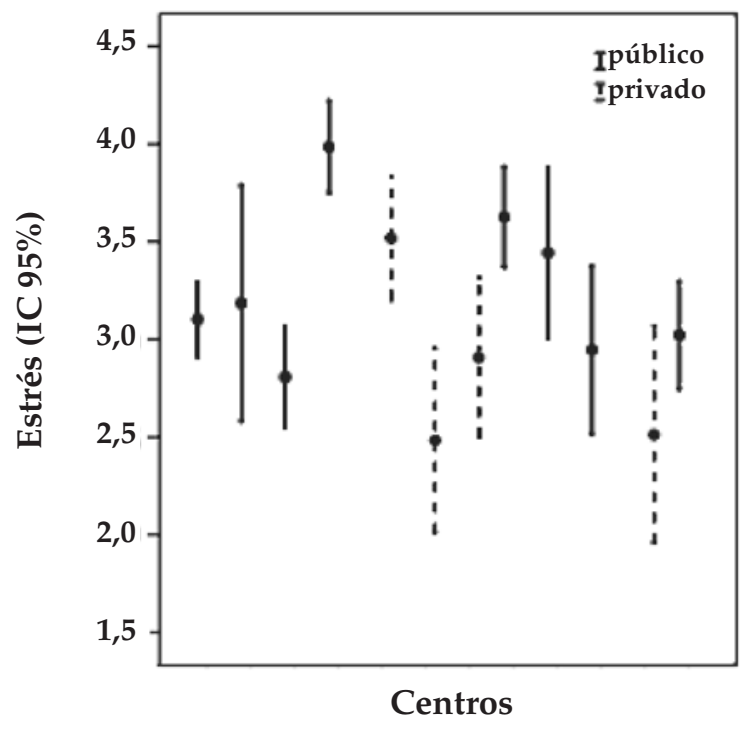

encontró la mayor puntuación para el estrés en la alteración del rol parental $(3,4)$. Los autores concluyen que la experiencia de las madres en las UCIN es altamente estresante, en particular relacionada con su marginalización de su rol parental.

Las madres presentaron un mayor nivel de estrés que los padres, lo cual es concordante con la mayoría de los estudios existentes. ${ }^{21,24}$ Las características de padres/madres que se asociaron a un mayor estrés parental en nuestro estudio fueron un menor nivel educacional, desempleo y aquellos padres que no habían tomado en brazos a su hijo.

Nuestros resultados son concordantes con un estudio que encontró que la adaptación materna a los aspectos estresantes de la hospitalización neonatal está fuertemente asociada al nivel socioeconómico, educacional y estatus laboral. ${ }^{25}$ Esto último podría explicarse por la menor disponibilidad de recursos para adaptarse a esta situación y a la dificultad para comprender la información recibida.

Otro estudio en madres de prematuros asoció un menor nivel educacional con la percepción de un menor apoyo recibido por parte de enfermería, mayor estrés y depresión materna. ${ }^{26}$ Otros estudios también han reportado que existe un mayor nivel de estrés en madres más jóvenes. ${ }^{19}$

En el presente estudio, los padres que habían tomado en brazos, al menos, una vez a su RN mostraron un menor nivel de estrés inicial. Este hallazgo es muy relevante y refuerza lo importante que es para los padres el contacto físico precoz con su hijo, tanto para reducir el estrés como para promover interacciones tempranas positivas que favorezcan un apego seguro. ${ }^{27,28}$ Esto es también consistente con estudios que sugieren que el contacto físico y la cercanía emocional entre la madre y su hijo son cruciales para el bienestar físico y emocional de ambos. ${ }^{29}$

Adicionalmente, nuestros resultados muestran que el factor que genera mayor estrés en los padres en relación con la condición de su hijo es el requerimiento de apoyo ventilatorio. Otros estudios han identificado, además del apoyo ventilatorio, el menor peso de nacimiento y la edad gestacional como factores estresantes para los padres, ${ }^{30}$ así como la percepción de qué tan enfermo se encuentra su hijo. ${ }^{31}$

El estrés parental fue mayor en los padres que se atienden en centros públicos respecto de los privados. Esto podría ser explicado 
por diferencias en las instalaciones, recursos y también por la mayor vulnerabilidad de la población. Un nivel socioeconómico bajo se ha identificado como de riesgo en el proceso de adaptación al nacimiento de un hijo prematuro, ya que puede limitar el acceso de la familia a recursos financieros, sociales y culturales. ${ }^{25}$

Este estudio tiene algunas limitaciones. Solo incluye la evaluación del período inicial de la hospitalización del RNMBPN y excluye a los RN que se consideraban de extrema gravedad. De acuerdo con otros estudios, el estrés parental puede continuar y prolongarse por varios meses en padres de prematuros extremos. ${ }^{9,10}$ Adicionalmente, no se obtuvo información acerca de otros factores que se han relacionado con el estrés parental, como lactancia materna, ansiedad y depresión. ${ }^{18,32}$

La mayor fortaleza de este estudio es que incluye un importante número de centros y provee información novedosa para la región sudamericana.

Este estudio también apunta a generar una mayor sensibilización en esta área y a poner mayor atención a los aspectos psicosociales de los padres de prematuros hospitalizados en la UCIN. Asimismo, refuerza la importancia de contar con maternidades centradas en la familia ${ }^{33} y$ un mayor involucramiento de profesionales del área de la piscología como parte del equipo de salud. Parece relevante que el personal de las UCIN identifique los factores causantes de estrés parental para que puedan desarrollar políticas de intervención que permitan manejar mejor las ansiedades de los padres, reducir su estrés, favorecer conductas de apego y estimular su habilidad para comprender y adaptarse al complejo entorno de las UCIN.

\section{CONCLUSIONES}

Tener un RNMBPN hospitalizado en la UCIN es una experiencia estresante para sus padres. El factor más relevante es la alteración en su rol parental. Se identifican varios factores asociados a mayor estrés parental, entre los cuales se destaca no haber tomado en brazos a su RN. El estrés parental fue mayor en madres que en padres y en centros públicos que en privados. Será importante realizar nuevos estudios en la región con intervenciones que ayuden a mitigar el estrés parental y que apunten a mejorar el bienestar emocional de los padres.

\section{Agradecimientos}

Agradecemos a todos los centros de la Red Neocosur que participaron en este estudio.

\section{REFERENCIAS}

1. Marshall G, Luque MJ, Gonzalez A, D’Apremont I, et al. Center variability in risk of adjusted length of stay for very low birth weight infants in the Neocosur South American Network. J Pediatr (Rio J) 2012;88(6):524-30.

2. Grandi C, González MA, Naddeo S, Basualdo MN, et al. Relación entre estrés psicosocial y el parto prematuro: una Investigación Interdisciplinaria en el área urbana de Buenos Aires. Rev Hosp Matern Infant Ramón Sardá 2008;27(2):51-69.

3. Ruiz AL, Ceriani Cernadas JM, Cravedi V, Rodríguez D. Estrés y depresión en madres de prematuros: un programa de intervención. Arch Argent Pediatr 2005;103(1):36-45.

4. Spear ML, Leef K, Epps S, Locke R. Family reactions during infants' hospitalization in the neonatal intensive care unit. Am J Perinatol 2002;19(4):205-13.

5. Muller-Nix C, Forcada-Guex M, Pierrehumbert B, Jaunin L, et al. Prematurity, maternal stress and mother-child interactions. Early Hum Dev 2004;79(2):145-58.

6. Forcada-Guex M, Borghini A, Pierrehumbert B, Ansermet F, et al. Prematurity, maternal posttraumatic stress and consequences on the mother-infant relationship. Early Hum Dev 2011;87(1):21-6.

7. Korja R, Latva R, Lehtonen L. The effects of preterm birth on mother-infant interaction and attachment during the infant's first two years. Acta Obstet Gynecol Scand 2012;91(2):164-73.

8. Morisod-Harari M, Borghini A, Hohlfeld P, Forcada-Guex $\mathrm{M}$, et al. Influence d'une hospitalisation prénatale sur les facteurs de stress parentaux lors d'une naissance prématurée. J Gynecol Obstet Biol Reprod (Paris) 2013;42(1):64-70.

9. Singer LT, Salvator A, Guo S, Collin M, et al. Maternal psychological distress and parenting stress after the birth of a very low-weight infant. JAMA 1999;281(9):799-805.

10. Treyvaud K, Doyle LW, Lee KJ, Roberts G, et al. Family functioning, burden and parenting stress 2 years after very preterm birth. Early Hum Dev 2011;87(6):427-31.

11. Huhtala M, Korja R, Lehtonen L, Haataja L, et al. Associations between parental psychological well-being and socio-emotional development in 5-year-old preterm children. Early Hum Dev 2014;90(3):119-24.

12. Red Neonatal Neocosur. [Acceso: 6 de abril de 2015]. Disponible en: www.neocosur.org.

13. Miles MS, Funk SG, Carlson J. Parental Stressor Scale: neonatal intensive care unit. Nurs Res 1993;42(3):148-52.

14. Oronoz B, Alonso-Arbiol I, Balluerka N. A Spanish adaptation of the Parental Stress Scale. Psicothema 2007;19(4):687-92.

15. Franck LS, Cox S, Allen A, Winter I. Measuring neonatal intensive care unit-related parental stress. J Adv Nurs 2005;49(5):608-15.

16. Reid T, Bramwell R. Using the Parental Stressor Scale: NICU with a British sample of mothers of moderate risk preterm infants. J Reprod Infant Psychol 2003;21(4):279-91.

17. Ichijima E, KirkR, Hornblow A. Parental support in neonatal intensive care units: a cross-cultural comparison between New Zealand and Japan. J Pediatr Nurs 2011;26(3):206-15.

18. Alkozei A, McMahon E, Lahav A. Stress levels and depressive symptoms in NICU mothers in the early postpartum period. I Matern Fetal Neonatal Med 2014;27(17):1738-43.

19. Montirosso R, Provenzi L, Calciolari G, Borgatti R. Measuring maternal stress perceived support in 25 Italian NICUs. Acta Paediatr 2012;101(2):136-42. 
20. Gonzalez MA. Stress, depresión, ansiedad en las madres de los prematuros internados. En: Sociedad Argentina de Pediatría. $1^{\circ}$ Congreso Argentino de Neonatología; 29 de septiembreal 2 de octubre de 2010; Buenos Aires, Argentina. [Acceso: 11 de abril de 2015]. Disponible en: http:/ / www. sap.org.ar/docs/congresos/2010/neo/gonzalezstress.pdf.

21. Miles MS, Funk SG, Kasper MA. The stress response of mothers and fathers of preterm infants. Res Nurs Health 1992;15(4):261-9.

22. Chourasia N, Surianarayanan $\mathrm{P}$, Bethou $\mathrm{A}$, Bhat V.Stressors of NICU mothers and the effect of counseling-experience from a tertiary care teaching hospital, India. J Matern Fetal Neonatal Med 2013;26(6):616-8.

23. Chourasia N, Surianarayanan P, Adhisivam B, Vishnu Bhat B. NICU admissions and maternal stress levels. Indian J Pediatr 2013;80(5):380-4.

24. Matricardi S, Agostino R, Fedeli C, Montirosso R. Mothers are not fathers: differences between parents in the reduction of stress levels after a parental intervention in a NICU. Acta Paediatr 2013;102(1):8-14.

25. Pinelli J. Effects of family coping and resources on family adjustment and parental stress in the acute phase of NICU experience. Neonatal Netw 2000;19(6):27-37.

26. Davis L, Edwards H, Mohay H, Wollin J. The impact of very premature birth on the psychological health of mothers. Early Hum Dev 2003;73(1-2):61-70.
27. Korja R, Maunu J, Kirjavainen J, Savonlahti E, et al. Motherinfant interaction is influenced by the amount of holding in preterm infants. Early Hum Dev 2008;84(4):257-67.

28. Sangüesa P, Farkas $C$, Rochet $P$. The relationship between maternal representations and interactive behaviour with the mother's emotional state, perceived social support and the infant's risk level in a group of premature babies. Estud Psicol 2014;35(1):80-103.

29. Flacking R, Lehtonen L, Thomson G, Axelin A, et al. Closeness and separation in neonatal intensive care. Acta Paediatr 2012;101(10):1032-7.

30. Meyer EC, Garcia Coll CT, Seifer R, Ramos A, et al. Psychological distress in mothers of preterm infants. J Dev Behav Pediatr 1995;16(6):412-7.

31. Shields-Poë D, Pinelli J. Variables associated with parental stress in neonatal intensive care units. Neonatal Netw 1997;16(1):29-37.

32. Lau C, Hurst NM, Smith EO, Schanler RJ. Ethnic/ racial diversity, maternal stress, lactation and very low birthweight infants. J Perinatol 2007;27(7):399-408.

33. Gooding JS, Cooper LG, Blaine AI, Franck LS, et al. Family support and family-centered care in the neonatal intensive care unit: origins, advances, impact. Semin Perinatol 2011;35(1):20-8.

"La creatividad es más que ser simplemente diferente.

Cualquiera puede hacer extravagancias, eso es fácil.

Lo difícil es ser tan simple como Bach." 